\title{
Non-Occlusive Mesenteric Ischemia After Resuscitative Endovascular Balloon Occlusion of the Aorta for Out-of-Hospital Cardiac Arrest due to Massive Gastrointestinal Bleeding
}

\author{
Shinsuke Tanizaki MD, Takeo Matsumoto MD, Misaki Murasaki MD, \\ Minoru Hayashi MD, Shigenobu Maeda MD and Hiroshi Ishida MD \\ Department of Emergency Medicine, Fukui Prefectural Hospital, Fukui, Japan
}

\begin{abstract}
Resuscitative endovascular balloon occlusion of the aorta (REBOA) has been used as a method of controlling intraabdominal bleeding in case of hemorrhagic shock and an adjunct to improve traditional advanced cardiac life support in non-traumatic cardiac arrest. Partial REBOA is proposed as an alternative method to regulate low-volume continuous blood flow across the area of occlusion with the aim of minimizing the risk of ischemia-reperfusion injury. An 82-year-old male suffered an out-of-hospital cardiac arrest due to massive gastric bleeding. He was initially resuscitated with partial REBOA but died of non-occlusive mesenteric ischemia (NOMI) or rebleeding. REBOA was performed during his cardiac arrest and deflated after the return of spontaneous circulation. We aimed for a proximal arterial pressure of $70-80 \mathrm{mmHg}$ and a distal arterial pressure of $20-30 \mathrm{mmHg}$. The total time of REBOA was $25 \mathrm{~min}$ of complete occlusion and $88 \mathrm{~min}$ of partial occlusion. The possible causes of NOMI were age of the patient, the low flow state with prolonged cardiopulmonary resuscitation, the lower proximal-to-distal gradient of the partial REBOA, and the longer duration of total occlusion. Further studies may be required to determine the optimal distal pressure during partial REBOA to limit the burden of mesenteric ischemia.
\end{abstract}

Keywords: Gastrointestinal Bleeding; Non-occlusive Mesenteric Ischemia; Out-of-Hospital Cardiac Arrest; Resuscitative Endovascular Balloon Occlusion of the Aorta

\section{BACKGROUND}

Resuscitative endovascular balloon occlusion of the aorta (REBOA) has been used to gain proximal aortic control and to decrease the distal hemorrhage until definitive hemorrhage control is achieved [1]. REBOA has also recently been used as an adjunct to improve traditional

\section{Corresponding author:}

Shinsuke Tanizaki, Department of Emergency Medicine, Fukui Prefectural Hospital, 2-8-1, Yotsui, Fukui 910-8526, Japan.

Email: sytanizak@yahoo.co.jp.

(c) 2020 CC BY 4.0 - in cooperation with Depts. of Cardiothoracic/ Vascular Surgery, General Surgery and Anesthesia, Örebro University Hospital and Örebro University, Sweden advanced cardiac life support in non-traumatic cardiac arrest (NTCA) [2,3]. Partial REBOA is proposed as an alternative method to regulate the continuous lowvolume blood flow across the area of occlusion with the aim of minimizing the risk of ischemia-reperfusion injury $[4,5]$. We present a case of a patient who suffered an out-of-hospital cardiac arrest due to massive gastric ulcer bleeding and underwent partial REBOA but died of non-occlusive mesenteric ischemia (NOMI), or rebleeding.

\section{CASE REPORT}

An 82-year-old male was found lying at home, unresponsive, and having passed a large amount of melena. He had been taking non-steroidal anti-inflammatory drugs for about two weeks because of a lumbar body 
fracture. He had a history of distal aortic arch aneurysm followed with non-surgical treatment and idiopathic pulmonary fibrosis. The emergency medical service (EMS) was called immediately. On the arrival of the EMS 20 min later, he was unresponsive and tachycardic without a radial pulse. Fluid resuscitation was initiated by paramedics. On the way to the hospital, after $11 \mathrm{~min}$ and about $1 \mathrm{~min}$ before arrival at the hospital, the patient went into pulseless electrical activity. Cardiopulmonary resuscitation (CPR) was immediately initiated by the paramedics. On arrival at the emergency department, he was in asystole. Advanced cardiac life support was performed (Figure 1). We suspected that the cardiac arrest was due to hemorrhagic shock from gastrointestinal (GI) bleeding because he had suffered massive melena. We speculated that if the GI bleeding could be stabilized with REBOA there would be a better chance of achieving definitive control of the bleeding and patient resuscitation. Endotracheal intubation was performed immediately and transfusion with type-O packed red blood cells (PRBCs) was commenced 5 min after arrival at the hospital. The right common femoral artery was punctured and a 7-French sheath was introduced. A Rescue Balloon-ER $®$ (Tokai Medical Products, Aichi, Japan) was inserted over the metal guidewire and placed in the supradiaphragmatic descending thoracic aorta. It was fully inflated with $25 \mathrm{~mL}$ of normal saline. After $2 \mathrm{~min}$ of occlusion of the aorta and 13 min of cardiac arrest, return of spontaneous circulation (ROSC) was achieved. The patient was administrated $3 \mathrm{mg}$ of adrenaline during the $13 \mathrm{~min}$ of CPR. Initial laboratory results were as follows: hemoglobin, $5.3 \mathrm{~g} / \mathrm{dL} ; \mathrm{pH}, 6.817$; lactate, $168 \mathrm{mg} / \mathrm{dL}$; and potassium, $5.5 \mathrm{mEq} / \mathrm{L}$. His proximal arterial pressure was monitored with a left radial arterial line, while his distal arterial pressure was monitored with a 5-French sheath in the left femoral artery.

Five minutes after initial ROSC, the patient went into ventricular fibrillation (VF) and CPR with defibrillation was performed. After 2 min of VF, ROSC was achieved for the second time. The patient's systolic blood pressure (SBP), monitored by proximal arterial pressure, reached $100 \mathrm{mmHg}$. After $10 \mathrm{~min}$ of REBOA at complete inflation, the balloon was completely deflated in a stepwise fashion over a period of $4 \mathrm{~min}$ to terminate aortic occlusion. However, his SBP gradually decreased to $55 \mathrm{mmHg}$ 10 min after complete deflation. The balloon was therefore partially re-inflated with $10 \mathrm{~mL}$ of normal saline and manual pressure pumping of the transfusion was performed. We aimed for a proximal arterial pressure of $70-80 \mathrm{mmHg}$ and a distal arterial pressure of 20-30 mmHg. Although his SBP gradually increased to $80 \mathrm{mmHg}$, VF occurred again after $12 \mathrm{~min}$ of partial REBOA. CPR was performed, and the balloon was again inflated completely. After 2 min of the second episode of VF, ROSC was achieved for the third time. The REBOA was converted to partial inflation with $10 \mathrm{~mL}$ of normal saline 15 min after the second complete REBOA. At this point, the laboratory results were as follows: hemoglobin, $6.6 \mathrm{~g} / \mathrm{dL}$; $\mathrm{pH}, 6.666$; lactate, $190 \mathrm{mg} / \mathrm{dL}$; and potassium, $5.3 \mathrm{mEq} / \mathrm{L}$. A truncal contrast-enhanced computed tomography (CT) scan was performed $77 \mathrm{~min}$ after arrival at the hospital. However, although gastroduodenal hemorrhage was observed, the bleeding source could not be identified. Since we observed a gastric hematoma without active extravasation of contrast, we concluded that active extravasation could not be detected via abdominal angiography. We decided to perform esophagogastroduodenoscopy (EGD) instead. EGD was performed in the intensive care unit (ICU) 96 min after arrival at our hospital. Multiple gastric A1-stage ulcers were detected in the antrum and angular notch of the lesser curvature (Figure 2a). Cauterization of an ulcer with visible vessels was performed with a Coagulasper Hemostatic Grasper. At 132 min after arrival at our hospital, the REBOA balloon was completely deflated. The patient's Glasgow coma scale score was E3VTM4 at this point. The laboratory results were as follows: hemoglobin, $7.9 \mathrm{~g} / \mathrm{dL}$; $\mathrm{pH}, 7.058$; lactate, $194 \mathrm{mg} / \mathrm{dL}$; and potassium, $4.6 \mathrm{mEq} / \mathrm{L}$. The total time of REBOA was $25 \mathrm{~min}$ of complete occlusion and 88 min of partial occlusion. The transfusion administered between arrival at hospital and the CT was 8 units of PRBCs and 2 units of fresh frozen plasma. The transfusion administered between CT and complete deflation of the balloon was 2 units of PRBCs, 8 units of fresh frozen plasma, and 10 units of platelets.

Twelve hours after complete deflation of REBOA, the patient's consciousness level was E4VTM6. He was extubated and the REBOA was removed. At this point, the laboratory results were as follows: hemoglobin, $15.4 \mathrm{~g} / \mathrm{dL}$; $\mathrm{pH}, 7.421$; and lactate, $66 \mathrm{mg} / \mathrm{dL}$. His SBP subsequently decreased to $53 \mathrm{mmHg}$ and a massive amount of dark blood came out through the nasogastric tube. Three hours after extubation, his respiration became agonal. He was reintubated and a transfusion was restarted. Although EGD was performed in the ICU again, no active hemorrhage was detected. After EGD, an abdominal contrast-enhanced CT scan was performed to detect the source of the bleeding. The CT scan showed a decreased contrast enhancement in the ileum and colon, presence of air in the colonic wall and the superior mesenteric vein, and enhancement in the superior mesenteric artery (Figure $2 b$ ). No aortoiliac injury or limb ischemia occurred. We concluded that NOMI, or rebleeding, was the cause of the second bout of hemodynamic instability. Further treatment was withheld at his family's request. Then, 14 hours after the second intubation, the patient died.

\section{Ethical Approval and Informed Consent}

Ethical approval to report these cases was given by Fukui Prefectural Hospital. Written informed consent was obtained from the patient's family. 


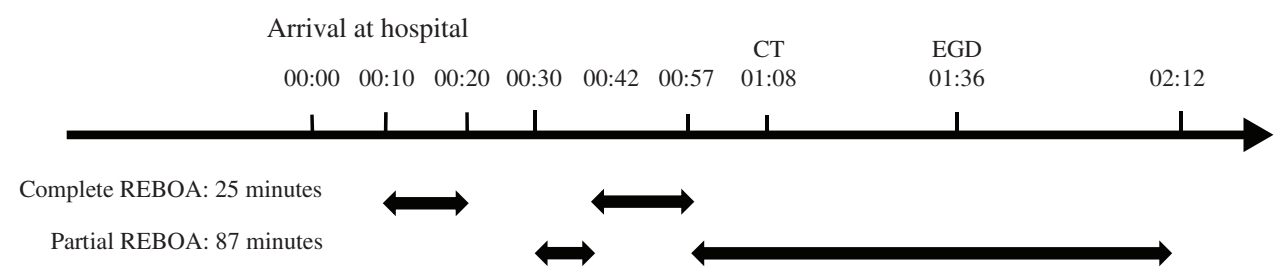

Figure 1 The clinical course of this patient. The horizon axis represents the elapsed time after arrival at hospital. REBOA: resuscitative endovascular balloon occlusion of the aorta; CT: computed tomography; EGD: esophagogastroduodenoscopy.
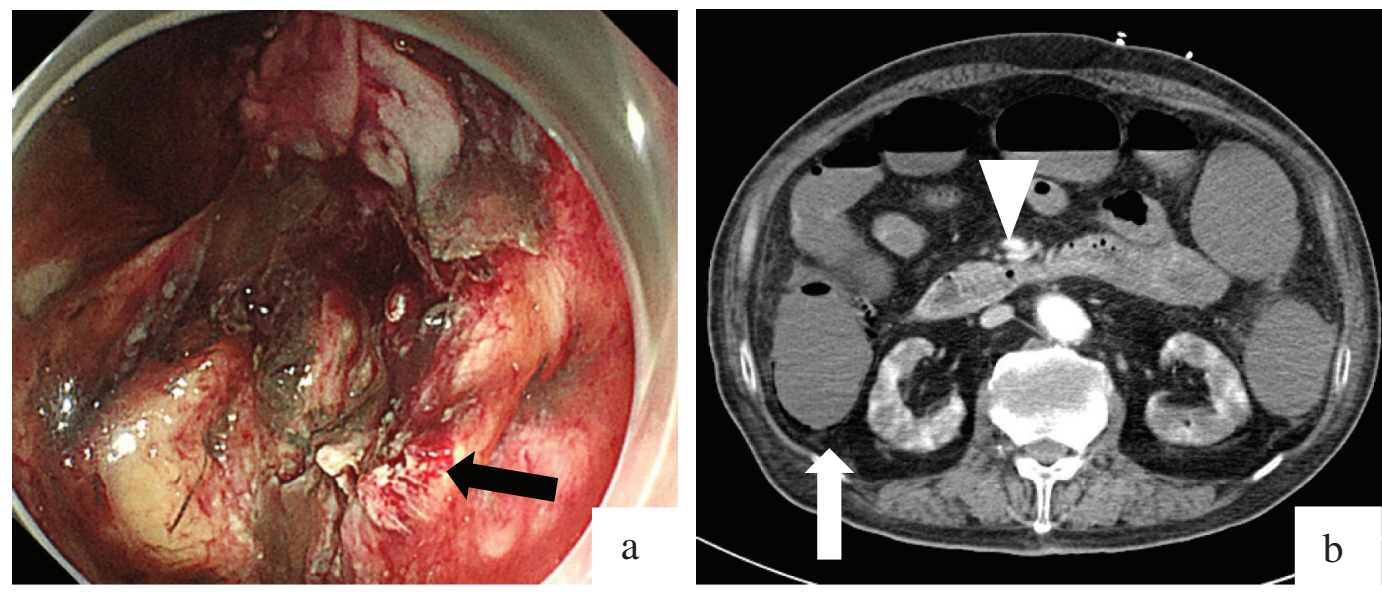

Figure 2 Diagnostic imaging of this patient. (a) First esophagogastroduodenoscopy. The black arrow indicates gastric A1-stage ulcers with visible vessels in the angular notch of the lesser curvature. (b) The abdominal contrast-enhanced computed tomography scan after reintubation. The white arrow indicates decreased contrast enhancement in the colon and air in the colonic wall. The white arrowhead indicates contrast enhancement in the superior mesenteric artery.

\section{DISCUSSION}

This case involved the use of partial REBOA during NTCA caused by a massive hemorrhage from gastric ulcers with initially successful resuscitation followed by fatal NOMI. Although there have been some reports on the use of REBOA in hemorrhagic shock caused by GI bleeding [1], there is currently no evidence of the use of REBOA to control massive GI bleeding in patients who are unresponsive to resuscitation or in cardiac arrest. Traditional transfusion without REBOA with permissive hypotension and definitive EGD to control the hemorrhage might not have been a better option because the patient in our case had two episodes of VF, regardless of the combination of REBOA and transfusion. Further research into the use of REBOA as a bridge is required.

Experimental studies have demonstrated that the occlusion of blood flow to the distal aorta in animal models of NTCA improved the hemodynamics of the heart and brain and increased rates of ROSC and shortterm survival [6-8]. The clinical literature involving human subjects with NTCA is limited to several case reports and case series [2,9]. This case demonstrated that REBOA is a feasible method to use during CPR for NTCA, to increase coronary and cerebral perfusion, and to achieve ROSC.

Partial REBOA is an alternative technique that may minimize the negative physiological consequences related to the profound distal ischemia caused by complete aortic occlusion. Clinical reports describing the use of partial REBOA have been limited to several case reports and case series. Overall, positive outcomes were reported $[4,5]$. The patient in our case developed NOMI within 19 hours but did not develop lower extremity ischemia. We targeted a left femoral arterial pressure of $20-30 \mathrm{mmHg}$, about $25-30 \%$ of proximal pressure, as an indicator of distal flow. We speculated that if we aimed for a distal pressure of around $40 \mathrm{mmHg}$ in this case, in which the patient would experience NTCA caused by severe hemorrhagic shock, there would be a lower chance of a proximal pressure of $80 \mathrm{mmHg}$. In our study, the partial REBOA with a $30 \%$ proximal-to-distal gradient was lower than the $50-70 \%$ that Russo et al. had, and had a greater chance of visceral ischemia [10]. Another possible cause of NOMI was the longer duration 
of total occlusion than that in a previous study (median: $58 \mathrm{~min}$ ) [5]. In addition, a previous report indicated that a longer duration of complete occlusion was associated with fasciotomy of the lower extremities [11]. In our case, the patient was also suffering from a vascular disease. This might have predisposed him to having a NOMI with reduced flow. In our case, NOMI could be secondary to the age of the patient and low flow state with prolonged CPR. Although the partial REBOA in our study had the lower proximal-to-distal gradient and the longer duration of total occlusion than those in the previous reports, it was unclear whether the REBOA was the direct cause of NOMI $[5,10]$. Further research into the available and reliable parameters for monitoring the mesenteric or bowel blood flow is required. In our case, the patient experienced rebleeding after extubation. Although no active gastroduodenal bleeding was detected on the second EGD, it was difficult to determine whether the patient died from NOMI or rebleeding.

The current management of acute upper GI bleeding begins with procedural intervention with endoscopy [12]. Early surgical treatment without endoscopy has been considered for patients with recurrent massive upper GI hemorrhage following initial endoscopy [13]. In our case, surgical intervention was not performed as the primary method of treating GI bleeding. Although our patient suffered three resuscitation attempts due to ongoing bleeding, operative intervention was not performed due to the age of the patient and multiple comorbidities, including a distal aortic arch aneurysm with non-surgical treatment and idiopathic pulmonary fibrosis. Based on the unsuccessful outcome with EGD, we speculated that exploratory laparotomy immediately after the third ROSC may have decreased the risk of NOMI and the REBOA time.

\section{CONCLUSION}

We achieved ROSC with REBOA during NTCA caused by massive GI bleeding. The outcome was eventually unsuccessful because of rebleeding or the development of NOMI. The possible causes of NOMI were age of the patient, the low flow state with prolonged cardiopulmonary resuscitation, the lower proximal-to-distal gradient of partial REBOA, and the longer duration of total occlusion.

\section{Ethics Statement}

(1) All the authors mentioned in the manuscript have agreed to authorship, read and approved the manuscript, and given consent for submission and subsequent publication of the manuscript.

(2) The authors declare that they have read and abided by the JEVTM statement of ethical standards including rules of informed consent and ethical committee approval as stated in the article.

\section{Conflicts of Interest}

The authors declare that they have no conflicts of interest.

\section{Funding}

The author(s) received no financial support for the research, authorship, and/or publication of this article.

\section{Author Contributions}

All authors have made substantive contributions to the study and manuscript writing.

\section{REFERENCES}

[1] Hoehn MR, Hansraj NZ, Pasley AM, et al. Resuscitative endovascular balloon occlusion of the aorta for non-traumatic intra-abdominal hemorrhage. Eur J Trauma Emerg Surg. 2019;45(4):713-8.

[2] Aslanger E, Golcuk E, Oflaz H, et al. Intraaortic balloon occlusion during refractory cardiac arrest. A case report. Resuscitation. 2009;80(2):281-3.

[3] Daley J, Morrison JJ, Sather J, Hile L. The role of resuscitative endovascular balloon occlusion of the aorta (REBOA) as an adjunct to ACLS in non-traumatic cardiac arrest. Am J Emerg Med. 2017;35(5): 731-6.

[4] Davidson AJ, Russo RM, DuBose JJ, Roberts J, Jurkovich GJ, Galante JM. Potential benefit of early operative utilization of low profile, partial resuscitative endovascular balloon occlusion of the aorta (P-REBOA) in major traumatic hemorrhage. Trauma Surg Acute Care Open. 2016;1(1):e000028.

[5] Matsumura Y, Matsumoto J, Kondo H, et al. Fewer REBOA complications with smaller devices and partial occlusion: evidence from a multicentre registry in Japan. Emerg Med J. 2017;34(12):793-9.

[6] Sesma J, Labandeira J, Sara MJ, Espila J, Arteche A, Saez MJ. Effect of intra-aortic occlusion balloon in external thoracic compressions during CPR in pigs. Am J Emerg Med. 2002;20(5):453-62.

[7] Gedeborg R, Rubertsson S, Wiklund L. Improved haemodynamics and restoration of spontaneous circulation with constant aortic occlusion during experimental cardiopulmonary resuscitation. Resuscitation. 1999;40(3): 171-80.

[8] Rubertsson S, Bircher NG, Alexander H. Effects of intra-aortic balloon occlusion on hemodynamics during, and survival after cardiopulmonary resuscitation in dogs. Crit Care Med. 1997;25(6):1003-9.

[9] Deakin CD, Barron DJ. Haemodynamic effects of descending aortic occlusion during cardiopulmonary resuscitation. Resuscitation. 1996;33(1):49-52.

[10] Russo RM, Williams TK, Grayson JK, et al. Extending the golden hour: partial resuscitative endovascular balloon occlusion of the aorta (P-REBOA) in a highly lethal liver injury model. J Trauma Acute Care Surg. 2016; 80(3):372-80. 
[11] Wasicek PJ, Teeter WA, Yang S, et al. Life over limb: Lower extremity ischemia in the setting of resuscitative endovascular balloon occlusion of the aorta (REBOA). Am Surg. 2018;84(6):971-7.

[12] Bardou M, Benhaberou-Brun D, Le Ray I, Barkun AN. Diagnosis and management of nonvariceal upper gastrointestinal bleeding. Nat Rev Gastroenterol Hepatol. 2012;9(2):97-104.

[13] Choy TY, Simoens C, Thill V, Mboti F, Vandaele S, Mendes da Costa P. Results of surgical treatment of uncontrollable upper gastrointestinal hemorrhage using endoscopy. Hepatogastroenterology. 2011;58(105):89-95. 